TRANSACTIONS OF THE

AMERICAN MATHEMATICAL SOCIETY

Volume 362, Number 12, December 2010, Pages 6325-6340

S 0002-9947(2010)04892-5

Article electronically published on July 9, 2010

\title{
MONGE-AMPÈRE MEASURES FOR CONVEX BODIES AND BERNSTEIN-MARKOV TYPE INEQUALITIES
}

\author{
D. BURNS, N. LEVENBERG, S. MA'U, AND SZ. RÉVÉSZ
}

\begin{abstract}
We use geometric methods to calculate a formula for the complex Monge-Ampère measure $\left(d d^{c} V_{K}\right)^{n}$, for $K \Subset \mathbb{R}^{n} \subset \mathbb{C}^{n}$ a convex body and $V_{K}$ its Siciak-Zaharjuta extremal function. Bedford and Taylor had computed this for symmetric convex bodies $K$. We apply this to show that two methods for deriving Bernstein-Markov type inequalities, i.e., pointwise estimates of gradients of polynomials, yield the same results for all convex bodies. A key role is played by the geometric result that the extremal inscribed ellipses appearing in approximation theory are the maximal area ellipses determining the complex Monge-Ampère solution $V_{K}$.
\end{abstract}

\section{INTRODUCTION}

For a function $u$ of class $C^{2}$ on a domain $\Omega \subset \mathbb{C}^{n}$, the complex Monge-Ampère operator applied to $u$ is

$$
\left(d d^{c} u\right)^{n}:=i \partial \bar{\partial} u \wedge \cdots \wedge i \partial \bar{\partial} u .
$$

For a plurisubharmonic (psh) function $u$ which is only locally bounded, $\left(d d^{c} u\right)^{n}$ is well-defined as a positive measure. Given a bounded set $E \subset \mathbb{C}^{n}$, we define the Siciak-Zaharjuta extremal function

$$
V_{E}(z):=\sup \left\{u(z): u \in L\left(\mathbb{C}^{n}\right), u \leq 0 \text { on } E\right\},
$$

where $L\left(\mathbb{C}^{n}\right)$ denotes the class of psh functions $u$ on $\mathbb{C}^{n}$ with $u(z) \leq \log ^{+}|z|+c(u)$. If $E$ is non-pluripolar, the upper-regularized function

$$
V_{E}^{*}(z):=\limsup _{\zeta \rightarrow z} V_{E}(\zeta)
$$

is a locally bounded psh function which satisfies $\left(d d^{c} V_{E}^{*}\right)^{n}=0$ outside of $\bar{E}$ and the total mass of $\left(d d^{c} V_{E}^{*}\right)^{n}$ is $(2 \pi)^{n}$.

In this paper we consider $E=K \subset \mathbb{R}^{n}$ to be a convex body, that is, a compact, convex set with non-empty interior. In this situation the function $V_{K}=V_{K}^{*}$ is continuous but it is not necessarily smooth, even if $K$ is smoothly bounded and

Received by the editors May 7, 2007 and, in revised form, July 4, 2008.

2010 Mathematics Subject Classification. Primary 32U15; Secondary 41A17, 32W20.

The first author was supported in part by NSF grants DMS-0514070 and DMS-0805877 (DB).

The fourth author was supported in part by the Hungarian National Foundation for Scientific Research, Project \#s K-72731 and K-81658 (SzR).

The third author was supported by a New Zealand Science and Technology Fellowship, contract no. IDNA0401 (SM).

This work was accomplished during the fourth author's stay in Paris under his Marie Curie fellowship, contract \# MEIF-CT-2005-022927. 
strictly convex. Indeed, for $K=\mathbb{B}_{\mathbb{R}}^{n}$, the unit ball in $\mathbb{R}^{n} \subset \mathbb{C}^{n}$, Lundin found 9], [1] that

$$
V_{K}(z)=\frac{1}{2} \log h\left(|z|^{2}+|z \cdot z-1|\right),
$$

where $|z|^{2}=\sum\left|z_{j}\right|^{2}, z \cdot z=\sum z_{j}^{2}$, and $h\left(\frac{1}{2}\left(t+\frac{1}{t}\right)\right)=t$, for $1 \leq t \in \mathbb{R}$. In this example, the Monge-Ampère measure $\left(d d^{c} V_{K}\right)^{n}$ has the explicit form

$$
\left(d d^{c} V_{K}\right)^{n}=n ! \operatorname{vol}(K) \frac{d x}{\left(1-|x|^{2}\right)^{\frac{1}{2}}}:=n ! \operatorname{vol}(K) \frac{d x_{1} \wedge \cdots \wedge d x_{n}}{\left(1-|x|^{2}\right)^{\frac{1}{2}}} .
$$

The main result of this paper is a general formula for this measure (see Theorem 4.1 and Corollary 4.5).

Theorem 1.1. Let $K$ be a convex body and $V_{K}$ its Siciak-Zaharjuta extremal function. The limit

$$
\delta_{B}^{K}(x, y)=\delta_{B}(x, y):=\lim _{t \rightarrow 0^{+}} \frac{V_{K}(x+i t y)}{t}
$$

exists for each $x \in K^{o}$ and $y \in \mathbb{R}^{n}$ and

$$
\left(d d^{c} V_{K}\right)^{n}=\lambda(x) d x \text {, where } \lambda(x)=n ! \operatorname{vol}\left(\left\{y: \delta_{B}(x, y) \leq 1\right\}^{*}\right) .
$$

Moreover, $\left(d d^{c} V_{K}\right)^{n}$ puts no mass on the boundary $\partial K$ (relative to $\mathbb{R}^{n}$ ).

Here, for a symmetric convex body $E$ in $\mathbb{R}^{n}$,

$$
E^{*}:=\left\{y \in \mathbb{R}^{n}: x \cdot y \leq 1, \text { for all } x \in E\right\}
$$

is also a symmetric convex body in $\mathbb{R}^{n}$, called the polar of $E$. The quantity $\delta_{B}(x, y)$ is continuous on $K^{o} \times \mathbb{R}^{n}$ and for each fixed $x \in K^{o}, y \rightarrow \delta_{B}(x, y)$ is a norm on $\mathbb{R}^{n}$; i.e., $\delta_{B}(x, y) \geq 0, \delta_{B}(x, \lambda y)=\lambda \delta_{B}(x, y)$ for $\lambda \geq 0$, and $\delta_{B}\left(x, y_{1}+y_{2}\right) \leq$ $\delta_{B}\left(x, y_{1}\right)+\delta_{B}\left(x, y_{2}\right)($ see $[3])$.

For a symmetric convex body, i.e., $K=-K$, Bedford and Taylor 3 showed the existence of the limit (1.2) and proved the formula (1.3) using the description of the Monge-Ampère solution given by Lundin [10. The present paper relies on the description of $V_{K}$ given in [6], 7] for general convex bodies $K$. [6] showed the existence, through each point $z \in \mathbb{C}^{n} \backslash K$, of a holomorphic curve on which $V_{K}$ is harmonic, while [7 showed that for many $K$ (all $K$ in $\mathbb{R}^{2}$ ) these curves give a continuous foliation of $\mathbb{C}^{n} \backslash K$ by holomorphic curves. It also showed that these curves are algebraic curves of degree 2 , and interpreted them in terms of a (finite dimensional) variational problem among real ellipses contained in $K$. The real points of such a quadratic curve describe an ellipse within $K$ of maximal area in its class of competitors. These competitor classes are specified by the points $c$ on the hyperplane $H$ at infinity in $\mathbb{P}^{n}$ through which the quadratic curves pass. The geometry of these foliations is our main tool.

The norm $\delta_{B}(x, y)$ is also related to Bernstein-Markov inequalities for real, multivariate polynomials on $K$. This will be explained in section 3, specifically, in equation (3.2) and the remarks after it. Conversely, a key role in the proof of the main result, Theorem 1.1, is played by the observation (Proposition 3.2) that the extremal inscribed ellipses appearing in a geometric approach (the "inscribed ellipse method" of Sarantopoulos [14, [13]) to Bernstein-Markov inequalities are the maximal area ellipses appearing in the determination of the Monge-Ampère solution as described above. A corollary of our main result is that the inscribed 
ellipse method and the pluripotential-theoretic method, due to Baran [1, 2] for obtaining Bernstein-Markov type estimates are equivalent for all convex bodies. This was straightforward for symmetric convex bodies. It was proved for simplices and conjectured for the general case as "Hypothesis A" in [12.

The remainder of the paper is organized as follows. In section 2 we recall in more detail some of the features of the leaf structure for the Monge-Ampère foliation. In section 3 we review the maximal inscribed ellipse problem from [14, [13] and its relation to Bernstein-Markov inequalities from approximation theory and to the Monge-Ampère maximal ellipses in section 2, We also sketch the relation to the extremal function $V_{K}$ for symmetric convex bodies [3, 2]. Finally, in section 4, using details of the Monge-Ampère foliation and its continuity, we prove the main results.

\section{REVIEW OF THE VARIATIONAL PROBLEM}

Let $K \subset \mathbb{R}^{n} \subset \mathbb{C}^{n}$ be a convex body, and consider $\mathbb{C}^{n} \subset \mathbb{P}^{n}$, the complex projective space with $H:=\mathbb{P}^{n} \backslash \mathbb{C}^{n}$ the hyperplane at infinity. Let $\sigma: \mathbb{P}^{n} \rightarrow \mathbb{P}^{n}$ be the anti-holomorphic map of complex conjugation, which preserves $\mathbb{C}^{n}$ and $H$, and is the identity on $\mathbb{R}^{n}$. Let $H_{\mathbb{R}}$ denote the real points of $H$ (fixed points of $\sigma$ in $H$ ). For any non-zero vector $c \in \mathbb{C}^{n}$, let $\sigma(c)=\bar{c}$, and let $[c] \in H$ be the point in $H$ given by the direction of $c$. If $[c] \neq[\bar{c}]$, then $c, \bar{c}$ span a complex subspace $V \subset \mathbb{C}^{n}$ of dimension two which is invariant under $\sigma$; hence $V$ is the complexification of a two-dimensional real subspace $V_{0} \subset \mathbb{R}^{n}$. If we translate $V$ by a vector $A \in \mathbb{R}^{n}$, we get a complex affine plane $V+A$ invariant by $\sigma$ and containing the real form $V_{0}+A$, the fixed points of $\sigma$ in $V+A$. Associated to the point $[c] \in H$, we consider holomorphic maps $f: \triangle \rightarrow \mathbb{P}^{n}, \triangle$ the unit disk in $\mathbb{C}$, such that $f(0)=[c]$ and $f(\partial \triangle) \subset K$. Such maps can be extended by Schwarz reflection to maps (still denoted) $f: \mathbb{P}^{1} \rightarrow \mathbb{P}^{n}$ by the formula

$$
f(\tau(\zeta))=\sigma(f(\zeta)) \in \mathbb{P}^{n},
$$

where $\tau: \mathbb{P}^{1} \rightarrow \mathbb{P}^{1}$ is the inversion $\tau(\zeta)=1 / \bar{\zeta}$. In particular, such maps have the form

$$
f(\zeta)=\rho \frac{C}{\zeta}+A+\rho \bar{C} \zeta,
$$

where $[c]=[C]$, i.e., $C=\lambda c$, for some $\lambda \in \mathbb{C}, A \in \mathbb{R}^{n}$, and $\rho>0$. Then $f\left(\mathbb{P}^{1}\right) \subset \mathbb{P}^{n}$ is a quadratic curve, and restricted to $\partial \triangle$, the unit circle in $\mathbb{C}, f$ gives a parametrization of a real ellipse inside the planar convex set $K \cap\left\{V_{0}+A\right\}$, with center at $A$. According to 7 , the extremal function $V_{K}$ is harmonic on the holomorphic curve $f(\triangle \backslash\{0\}) \subset \mathbb{C}^{n} \backslash K$ if and only if the area of the ellipse bounded by $f(\partial \triangle)$ is maximal among all those of the form (2.2).

For a fixed, normalized $C$, this is equivalent to varying $A \in \mathbb{R}^{n}$ and $\rho>0$ among the maps in (2.2) with $\mathcal{E}=f(\partial \triangle) \subset K$ in order to maximize $\rho$. Fixing $C$ amounts to prescribing the orientation (major and minor axis) and eccentricity of a family of inscribed ellipses in $K$. We will call an extremal ellipse $\mathcal{E}$ a maximal area ellipse, or simply $a$-maximal. In the case where $\partial K$ contains no parallel faces, for each $[c] \in H$ there is a unique $a$-maximal ellipse (Theorem 7.1, [7]); we denote the corresponding map by $f_{c}$. In this situation, the collection of complex ellipses $\left\{f_{c}(\triangle \backslash\{0\}):[c] \in H\right\}$ form a continuous foliation of $\mathbb{C}^{n} \backslash K$. In simple terms, this means that if $z, z^{\prime}$ are distinct points in $\mathbb{C}^{n} \backslash K$, with $\left|z-z^{\prime}\right|$ small, lying on 
leaves $L(z):=f_{c}(\triangle \backslash\{0\})$ and $L^{\prime}(z):=f_{c^{\prime}}(\triangle \backslash\{0\})$, then the corresponding leaf parameters in (2.2) are close; i.e., $C \sim C^{\prime}, \rho \sim \rho^{\prime}$ and $A \sim A^{\prime}$ (and of course $\left|\zeta_{z}\right| \sim\left|\zeta_{z^{\prime}}\right|$, where $f_{c}\left(\zeta_{z}\right)=z$ and $\left.f_{c^{\prime}}\left(\zeta_{z^{\prime}}\right)=z^{\prime}\right)$. Any convex body in $\mathbb{R}^{2}$ admits a continuous foliation; this follows by using ideas in [7. Moreover, if we let $\mathcal{C}$ denote the set of all convex bodies $K \subset \mathbb{R}^{n}$ admitting a continuous foliation, then $\mathcal{C}$ is dense in the Hausdorff metric in the set $\mathcal{K}$ of all convex bodies $K \subset \mathbb{R}^{n}$. This follows, for example, from the fact that strictly convex bodies $K$ belong to $\mathcal{C}$ (cf., Theorem 7.1 of [7]). In addition, all symmetric convex bodies admit a continuous foliation.

For convenience, instead of using the holomorphic curves $f(\triangle \backslash\{0\})$ we will work with the holomorphic curve $f(\mathbb{C} \backslash \bar{\triangle})$; thus $V_{K}$ being harmonic on this curve means that

$$
V_{K}(f(\zeta))=\log |\zeta| \text { for }|\zeta| \geq 1
$$

\section{INSCRIBED ELLIPSE PROBLEM}

Let $K \subset \mathbb{R}^{n}$ be a convex body. Consider the following geometric problem: fix $x \in K^{o}$ and a non-zero vector $y \in \mathbb{R}^{n}$ and consider all ellipses $\mathcal{E}$ lying in $K$ which contain $x$ and have a tangent at $x$ in the direction $y$. We write $y \in T_{x} \mathcal{E}$. That is, $\mathcal{E}=\mathcal{E}_{b}=\mathcal{E}_{b}(x, y)$ is given by a parameterization

$$
\theta \rightarrow r(\theta):=a \cos \theta+b y \sin \theta+(x-a)
$$

where $a \in \mathbb{R}^{n}$ and $b \in \mathbb{R}^{+}$are such that $r(\theta) \in K$ for all $\theta$. The problem is to maximize $b$ among all such ellipses. We will call such an ellipse a maximal inscribed ellipse (for $x, y$ ) or simply $b$-maximal. Note that $r(0)=x$ and $r^{\prime}(0)=b y$; thus one is allowed to vary $a$ and $b$ in (3.1). Often we will normalize and assume that $y$ is a unit vector. An observation which will be used later is that if we fix $a$, then $\mathcal{E}_{b}$ lies "inside" $\mathcal{E}_{b^{\prime}}$ if $b<b^{\prime}$ with two common points $x$ and $x-2 a$.

We give some motivation for studying this problem; this goes back to Sarantopolous (cf. [14] or [13]). For any such ellipse $\mathcal{E}$, if $p$ is a polynomial of $n$ real variables of degree $d$, say, with $\|p\|_{K} \leq 1$, then $t(\theta):=p(r(\theta))$ is a trigonometric polynomial of degree at most $d$ with $\|t\|_{[0,2 \pi]} \leq\|p\|_{K} \leq 1$ (since $\mathcal{E} \subset K$ ). By the Bernstein-Szegö inequality for trigonometric polynomials,

$$
\frac{\left|t^{\prime}(\theta)\right|}{\sqrt{\|\left. t\right|_{[0,2 \pi]} ^{2}-t(\theta)^{2}}} \leq d \text {. }
$$

From the chain rule,

$$
\left|t^{\prime}(0)\right|=\left|\nabla p(x) \cdot r^{\prime}(0)\right|=\left|D_{b y} p(x)\right|=b\left|D_{y} p(x)\right| .
$$

Thus

$$
b\left|D_{y} p(x)\right|=\left|t^{\prime}(0)\right| \leq d \sqrt{\left.|| t\right|_{[0,2 \pi]} ^{2}-t(0)^{2}} \leq d \sqrt{1-p(x)^{2}} ;
$$

i.e.,

$$
\frac{1}{\operatorname{deg} p} \frac{\left|D_{y} p(x)\right|}{\sqrt{1-p(x)^{2}}} \leq \frac{1}{b} .
$$

The left-hand side is related to what we shall refer to as a Bernstein-Markov inequality 11 it relates the directional derivative of $p$ at $x$ in the direction $y$ with the

\footnotetext{
${ }^{1}$ For any univariate algebraic polynomial of degree not exceeding $n$, the sharp uniform estimate for the derivative $\left\|p^{\prime}\right\|_{\infty,[-1,1]} \leq n\|p\|_{\infty,[-1,1]}$ is due to Markov, while the pointwise estimate
} 
sup-norm of $p$ on $K$ (the " 1 " on the right-hand side of (3.2) ) and the degree of $p$. This motivates the definition of the Bernstein-Markov pseudometric (cf. [5]): given $x \in K, y \in \mathbb{R}^{n}$, let

$$
\delta_{M}^{K}(x ; y)=\delta_{M}(x ; y):=\sup _{\|p\|_{K} \leq 1, \operatorname{deg} p \geq 1} \frac{1}{\operatorname{deg} p} \frac{\left|D_{y} p(x)\right|}{\sqrt{1-p(x)^{2}}} .
$$

(This definition makes sense for general compacta in $\mathbb{R}^{n}$.) Inequality (3.2) says that whenever you have an inscribed ellipse $\mathcal{E}_{b}=\mathcal{E}_{b}(x, y)$ through $x$ with tangent at $x$ in the direction of $y$, the number $1 / b$ gives an upper bound on the Bernstein-Markov pseudometric:

$$
\delta_{M}(x ; y) \leq 1 / b .
$$

Let

$$
b^{*}(x, y):=\sup \left\{b: \mathcal{E}_{b}(x, y) \subset K\right\} .
$$

Note that $b^{*}(x, t y)=b^{*}(x, y) / t$ for $t>0$. In the symmetric case, this is intimately related to $V_{K}$ :

Proposition 3.1. If $K=-K$, then $\delta_{M}(x ; y)=\frac{1}{b^{*}(x, y)}$. Moreover,

$$
\delta_{M}(x ; y)=\lim _{t \rightarrow 0^{+}} \frac{V_{K}(x+i t y)}{t} .
$$

As in the Introduction, define

$$
\delta_{B}^{K}(x ; y)=\delta_{B}(x, y):=\lim _{t \rightarrow 0^{+}} \frac{V_{K}(x+i t y)}{t},
$$

provided this limit exists. For symmetric convex bodies $K$, the proposition says that the limit does exist and we have

$$
\delta_{B}(x, y)=\delta_{M}(x, y)=\frac{1}{b^{*}(x, y)} .
$$

Moreover, for each fixed $x \in K^{o}$, the function $y \rightarrow \frac{1}{b^{*}(x, y)}$ is a norm (cf. Proposition 3.3). A proof of the existence of the limit was given by Bedford and Taylor [3. We sketch an alternate proof due to Baran [2].

Step 1. $\left.V_{K}(z)=\sup \{\log \mid h(z \cdot Z)) \mid: Z \in K^{*}\right\}$, where $h(w)=w+\sqrt{w^{2}-1}$ is the standard Joukowski map and

$$
K^{*}:=\{Z: x \cdot Z \leq 1 \text { for all } x \in K\}
$$

is the polar of $K$ (cf. [10], or [2, Proposition 1.15).

Step 2. We have the following explicit estimates on $h$ : if $|\alpha|<1,|\beta| \leq \sqrt{1-|\alpha|}$, and $0<\epsilon \leq 1 / 2$, then

$$
(1-\epsilon) \frac{|\beta|}{\sqrt{1-\alpha^{2}}} \leq \frac{1}{\epsilon} \log |h(\alpha+i \epsilon \beta)| \leq \frac{|\beta|}{\sqrt{1-\alpha^{2}}}
$$

(the inequality on the right-hand side is valid without the restriction $|\beta| \leq \sqrt{1-|\alpha|}$; cf. [2, Proposition 1.13). This states precisely that $\log |h|$ is Lipschitz as you approach $(-1,1)$ vertically and the Lipschitz constant grows like one-over-the-distance to the boundary points.

$\left|p^{\prime}(x)\right| \sqrt{1-x^{2}} \leq n\|p\|_{\infty,[-1,1]}$ is known as Bernstein's Inequality; see e.g. 4], pages 232-233. In approximation theory, these types of derivative estimates (or, in the multivariate case, gradient and directional derivative estimates) are usually termed Bernstein and/or Markov type inequalities. 
Now fix $x \in K^{o}$ and $y \in \mathbb{R}^{n}$; then for any $Z \in K^{*}$ and for $t>0$ small, since $(x+i t y) \cdot Z=x \cdot Z+i t y \cdot Z$,

$$
(1-\epsilon) \frac{1}{t} \frac{t|y \cdot Z|}{\sqrt{1-(x \cdot Z)^{2}}} \leq \frac{1}{t} \log |h((x+i t y) \cdot Z)| \leq \frac{1}{t} \frac{t|y \cdot Z|}{\sqrt{1-(x \cdot Z)^{2}}} .
$$

This gives

$$
\lim _{t \rightarrow 0^{+}} \frac{V_{K}(x+i t y)}{t}=\sup \left\{\frac{|y \cdot Z|}{\sqrt{1-(x \cdot Z)^{2}}}: Z \in K^{*}\right\} .
$$

To relate this with $b^{*}(x ; y)$, in the symmetric case, the $b$-maximal ellipse is easily seen to be an $a$-maximal ellipse (see the next proposition for a generalization of this), and the linear polynomial $p$ that maps the support "strip" of this ellipse to $[-1,1]$ (i.e., it maps one parallel support hyperplane to -1 and the other to +1 ) is easily seen to give

$$
\frac{1}{\operatorname{deg} p} \frac{\left|D_{y} p(x)\right|}{\sqrt{1-p(x)^{2}}}=\frac{1}{b^{*}(x, y)}
$$

so that we have the equality $\delta_{M}(x, y)=\frac{1}{b^{*}(x, y)}$. Thus, the first part of the proposition is proved. Moreover, we have the following formula for $b^{*}(x, y)$ :

$$
b^{*}(x ; y)=\inf \left\{\frac{\sqrt{1-(x \cdot w)^{2}}}{|y \cdot w|}: w \in K^{*}\right\} .
$$

To see this, in the symmetric case one considers symmetric ellipses in (3.1), i.e., $a:=x$, and, from the definition of $b^{*}(x ; y)$ and $K^{*}$ we can write

$$
\begin{aligned}
b^{*}(x ; y) & =\sup \left\{b: \sup _{w \in K^{*}, t \in[0,2 \pi]}|x \cos t \cdot w+y b \sin t \cdot w|=1\right\} \\
& =\sup \left\{b: \sup _{w \in K^{*}}\left[(w \cdot x)^{2}+b^{2}(w \cdot y)^{2}\right]=1\right\} .
\end{aligned}
$$

Basically unwinding things shows that this is the reciprocal of (3.7). For details we refer the reader to [11] or [5].

A key geometric observation which will be used in the next section is the following.

Proposition 3.2. For any convex body $K, a b$-maximal ellipse $\mathcal{E}$ is also an a-maximal ellipse.

Proof. First observe that an $a$-maximal ellipse $\mathcal{E}$ is characterized by the property that no translate of $\mathcal{E}$ lies entirely in the interior $K^{o}$ of $K$. For if $\mathcal{E}+v \subset K^{o}$ for some $v \neq 0$, one can dilate $\mathcal{E}+v$ to get an ellipse with the same orientation and eccentricity as $\mathcal{E}$ which lies in $K$ but has larger area. Conversely, if $\mathcal{E}$ is not an $a$-maximal ellipse, then one can find an ellipse $\mathcal{E}^{\prime}$ with the same orientation and eccentricity as $\mathcal{E}$ which lies in $K$ but has larger area. The convex hull $H$ of $\mathcal{E} \cup \mathcal{E}^{\prime}$ lies in $K$ and we can translate $\mathcal{E}$ within $H$ to an ellipse $\mathcal{E}^{\prime \prime}$ lying in the two-dimensional surface $S\left(\mathcal{E}^{\prime}\right)$ determined by $\mathcal{E}^{\prime}$; if $\mathcal{E}^{\prime \prime}$ does not lie in the "interior" of $S\left(\mathcal{E}^{\prime}\right)$, we simply translate it within this surface (since the area of $\mathcal{E}^{\prime}$ is greater than that of $\left.\mathcal{E}^{\prime \prime}\right)$ until it does.

Indeed, we need a slightly more precise statement: $\mathcal{E}$ is not an $a$-maximal ellipse if and only if there is a unit vector $v$ and $\delta>0$ such that $\mathcal{E}+s v \subset K^{o}$ for $0<s<\delta$, i.e., all translates by a small amount in some direction stay in $K^{o}$. This follows 
from the previous paragraph if we observe the following fact: if $K$ is a convex body, $u \in K$ and $u+v \in K^{o}$, then the entire half-open segment $(u, u+v]$ lies in $K^{o}$.

Suppose that $\mathcal{E}$ given by

$$
\theta \rightarrow a \cos \theta+b y \sin \theta+(x-a)
$$

is a $b$-maximal ellipse for $x, y$. For the sake of obtaining a contradiction, we assume that $\mathcal{E}$ is not an $a$-maximal ellipse. By the previous paragraph, we can find a nonzero vector $v$ and $\delta>0$ so that $\mathcal{E}_{s}:=\mathcal{E}+s v$ lies in $K^{o}$ for $0<s<\delta$. For $0<\epsilon<\delta / 2$, consider the ellipse $\tilde{\mathcal{E}}(\epsilon)$ given by

$$
r_{\epsilon}(\theta)=(a-\epsilon v) \cos \theta+b y \sin \theta+x-(a-\epsilon v) .
$$

We claim that $\tilde{\mathcal{E}}(\epsilon) \subset K^{o}$. Assuming this is the case, note that $r_{\epsilon}(0)=x \in \tilde{\mathcal{E}}(\epsilon)$ and $r_{\epsilon}^{\prime}(0)=b y$; in particular, the " $b$ " for $\tilde{\mathcal{E}}(\epsilon)$ is the same as the " $b$ " for $\mathcal{E}$. Since $\tilde{\mathcal{E}}(\epsilon) \subset K^{o}$, we can modify $\tilde{\mathcal{E}}(\epsilon)$ to an ellipse $\tilde{\mathcal{E}}(\epsilon)^{\prime}$ containing $x$ and lying in $K$ by replacing $b$ in (3.8) by $b^{\prime}>b$, contradicting the assumption that $\mathcal{E}$ is a $b$-maximal ellipse for $x, y$.

To verify that $\tilde{\mathcal{E}}(\epsilon) \subset K^{o}$, observe that for each fixed $\theta$, the point

$$
\begin{aligned}
& (a-\epsilon v) \cos \theta+b y \sin \theta+x-(a-\epsilon v) \\
= & a \cos \theta+b y \sin \theta+(x-a)+\epsilon v(1-\cos \theta)
\end{aligned}
$$

on $\tilde{\mathcal{E}}(\epsilon)$ lies on the ellipse $\mathcal{E}_{s_{\theta}}:=\mathcal{E}+\epsilon(1-\cos \theta) v$, where $s_{\theta}=\epsilon(1-\cos \theta) \leq 2 \epsilon<\delta$. Thus $\tilde{\mathcal{E}}(\epsilon) \subset K^{o}$.

For use in the next section, we prove some results about the function $b^{*}(x, y)$.

Proposition 3.3. For a convex body $K \subset \mathbb{R}^{n}, b^{*}(x, y)$ defined in (3.3) is a continuous function of $x \in K^{o}$ and $y \in \mathbb{R}^{n}$. Moreover, for each fixed $x \in K^{o}$, $y \rightarrow 1 / b^{*}(x, y)$ is a norm in $\mathbb{R}^{n}$.

Proof. For the continuity of $b^{*}(x, y)$, we first verify the uppersemicontinuity of this function. Fix a convex body $K$ and fix $x \in K^{o}$ and $y \in \mathbb{R}^{n}$. Let $\left\{x_{j}\right\} \subset K^{o}$ with $x_{j} \rightarrow x$ and $\left\{y_{j}\right\} \subset \mathbb{R}^{n}$ with $y_{j} \rightarrow y$. Let

$$
r_{j}(\theta)=a_{j} \cos \theta+b^{*}\left(x_{j}, y_{j}\right) y_{j} \sin \theta+\left(x_{j}-a_{j}\right)
$$

parameterize a $b$-maximal ellipse $\mathcal{E}_{j}$ for $K$ through $x_{j}$ in the direction $y_{j}$. Take a subsequence $\left\{j_{k}\right\}$ of positive integers so that the numbers $\left\{b^{*}\left(x_{j_{k}}, y_{j_{k}}\right)\right\}$ converge to a number $\tilde{b}$; and take a further subsequence (which we still call $\left\{j_{k}\right\}$ ) so that the vectors $\left\{a_{j_{k}}\right\} \subset \mathbb{R}^{n}$ converge to $a \in \mathbb{R}^{n}$. Consider the ellipse $\mathcal{E}$, where

$$
r(\theta)=a \cos \theta+\tilde{b} y \sin \theta+(x-a) .
$$

Since $x_{j_{k}} \rightarrow x, y_{j_{k}} \rightarrow y, b^{*}\left(x_{j_{k}}, y_{j_{k}}\right) \rightarrow \tilde{b}$ and $a_{j_{k}} \rightarrow a$, the functions $r_{j_{k}}$ converge uniformly to $r$ (equivalently, the ellipses $\mathcal{E}_{j_{k}}$ converge in the Hausdorff metric to $\mathcal{E})$. Thus $\mathcal{E}$ is an inscribed ellipse for $K$ through $x$ in the direction of $y$; hence $\tilde{b} \leq b^{*}(x, y)$, i.e.,

$$
\limsup _{x^{\prime} \rightarrow x, y^{\prime} \rightarrow y} b^{*}\left(x^{\prime}, y^{\prime}\right) \leq b^{*}(x, y) .
$$

To verify the lowersemicontinuity of $b^{*}(x, y)$, we fix $x \in K^{o}, y \in \mathbb{R}^{n}$ and $b^{\prime}<$ $b^{*}(x, y)$, and we show there is a $\delta>0$ such that for all $\left|x^{\prime}-x\right|<\delta,\left|y^{\prime}-y\right|<\delta$ there is an inscribed ellipse $\mathcal{E}^{\prime}$ through $x^{\prime}$ with tangent direction $y^{\prime}$ of the form

$$
\theta \rightarrow a^{\prime} \cos \theta+b^{\prime} y^{\prime} \sin \theta+\left(x^{\prime}-a^{\prime}\right) .
$$


Let $\mathcal{E}$ be a $b$-maximal ellipse through $x$ in the direction $y$ given by

$$
r(\theta)=a \cos \theta+b^{*}(x, y) y \sin \theta+(x-a) .
$$

If $x-2 a \in K^{o}$, then for $b^{\prime}<b$ the ellipse $\mathcal{E}_{b^{\prime}}$,

$$
r_{b^{\prime}}(\theta)=a \cos \theta+b^{\prime} y \sin \theta+(x-a),
$$

lies fully in $K^{o}$ (for $\mathcal{E}_{b^{\prime}}$ lies entirely "inside" of $\mathcal{E}$ except for the common points $x, x-2 a$, and $x \in K^{o}$ ). Then any sufficiently small translation $\mathcal{E}^{\prime}$,

$$
\theta \rightarrow a \cos \theta+b^{\prime} y \sin \theta+\left(x^{\prime}-a\right)
$$

of $\mathcal{E}_{b^{\prime}}$ by $x^{\prime}-x$, keeps $\mathcal{E}^{\prime}$ in $K^{o}$; hence replacing $y$ by $y^{\prime}$ sufficiently close to $y$ yields $\mathcal{E}^{\prime \prime}$

$$
\theta \rightarrow a \cos \theta+b^{\prime} y^{\prime} \sin \theta+\left(x^{\prime}-a\right)
$$

in $K$.

If $x-2 a \notin K^{o}$, we first modify $\mathcal{E}_{b^{\prime}}$ to $\mathcal{E}_{b^{\prime}, a^{\prime}}$ :

$$
r_{b^{\prime}, a^{\prime}}(\theta)=a^{\prime} \cos \theta+b^{\prime} y \sin \theta+\left(x-a^{\prime}\right)
$$

with $a^{\prime}-a=\delta(a-x)$ with $\delta>0$ sufficiently small so that $\mathcal{E}_{b^{\prime}, a^{\prime}} \subset K^{o}$. This is possible since the vectors

$$
r_{b^{\prime}, a^{\prime}}(\theta)-r_{b^{\prime}}(\theta)=\left(a-a^{\prime}\right)(1-\cos \theta)=\delta(1-\cos \theta)(x-a)
$$

point in the same direction for all $\theta$. Note that

$$
r_{b^{\prime}, a^{\prime}}(0)=x \text { and } r_{b^{\prime}, a^{\prime}}^{\prime}(0)=b^{\prime} y
$$

so that once again any sufficiently small translation $\mathcal{E}^{\prime}$,

$$
\theta \rightarrow a^{\prime} \cos \theta+b^{\prime} y \sin \theta+\left(x^{\prime}-a^{\prime}\right)
$$

of $\mathcal{E}_{b^{\prime}, a^{\prime}}$ by $x^{\prime}-x$, keeps $\mathcal{E}^{\prime}$ in $K^{o}$. Again, replacing $y$ by $y^{\prime}$ sufficiently close to $y$ yields $\mathcal{E}^{\prime \prime}$,

$$
\theta \rightarrow a^{\prime} \cos \theta+b^{\prime} y^{\prime} \sin \theta+\left(x^{\prime}-a^{\prime}\right)
$$

in $K$. This completes the proof that $(x, y) \rightarrow b^{*}(x, y)$ is continuous.

To show that $y \rightarrow 1 / b^{*}(x, y)$ is a norm, observe that $b^{*}(x, \lambda y)=\frac{1}{\lambda} b^{*}(x, y)$ for $\lambda>0$ and $0<b^{*}(x, y)<+\infty$ if $y \neq 0 \in \mathbb{R}^{n}$. Thus $b^{*}(x, 0)=+\infty$ so that $1 / b^{*}(x, y) \geq 0$ with equality precisely when $y=0$, and $1 / b^{*}(x, \lambda y)=\lambda / b^{*}(x, y)$ for $\lambda \geq 0$. To verify subadditivity in $y$, fix $x \in K^{o}$ and $y_{1}, y_{2} \in \mathbb{R}^{n}$. Let $\mathcal{E}_{1}$ and $\mathcal{E}_{2}$ be $b$-maximal ellipses through $x$ in the directions $y_{1}$ and $y_{2}$ as in (3.1):

$$
\theta \rightarrow r_{j}(\theta):=a_{j} \cos \theta+b_{j} y_{j} \sin \theta+\left(x-a_{j}\right), j=1,2
$$

here, $b_{j}:=b^{*}\left(x, y_{j}\right)$. Consider the convex combination

$$
\frac{b_{2}}{b_{1}+b_{2}} r_{1}(\theta)+\frac{b_{1}}{b_{1}+b_{2}} r_{2}(\theta) \text {. }
$$

By convexity, this ellipse, through $x$ in the direction $y_{1}+y_{2}$, lies in $K$ so that

$$
b^{*}\left(x, y_{1}+y_{2}\right) \geq \frac{b_{1} b_{2}}{b_{1}+b_{2}} .
$$

Taking reciprocals gives the desired result. 
For future use, we mention that in $\mathbb{R}^{2}$, if $\mathcal{E}$ is an $a$-maximal ellipse for $K$, then either

(1) $\mathcal{E} \cap \partial K$ contains exactly two points $a_{1}, a_{2}$, in which case the tangent lines to $\mathcal{E}$ at $a_{1}, a_{2}$ are parallel and determine a strip $S$ containing $K$ and $\mathcal{E}$ is an $a$-maximal ellipse for any rectangular truncation $T$ of $S$ with $\mathcal{E} \subset K \subset T$; or

(2) $\mathcal{E} \cap \partial K$ contains $m \geq 3$ points $a_{1}, \ldots, a_{m}$, in which case either a subset of three points from $\left\{a_{1}, \ldots, a_{m}\right\}$ can be found so that the tangent lines to $\mathcal{E}$ at these three points bound a triangle $T$ containing $K$ and $\mathcal{E}$ is an $a$-maximal ellipse for $T$, or a rectangular truncation $T$ of a strip $S$ with $\mathcal{E} \subset K \subset T$ can be found so that $\mathcal{E}$ is an $a$-maximal ellipse for $T$.

\section{MAin RESUlT}

For any compact set $K \subset \mathbb{R}^{n}$ with non-empty interior, take $x \in K^{o}$ and $y \in$ $\mathbb{R}^{n} \backslash\{0\}$. Then we always have the pointwise inequality

$$
\delta_{M}(x, y) \leq \delta_{B}^{(i)}(x, y):=\liminf _{t \rightarrow 0^{+}} \frac{V_{K}(x+i t y)}{t} .
$$

This follows from Proposition 2.1 in [5]. In particular, this inequality holds for any convex body $K$, with equality in case $K$ is symmetric (as we saw in the previous section). In this section, for $K$ convex, we prove that the $\operatorname{limit}_{t \rightarrow 0^{+}} \frac{V_{K}(x+i t y)}{t}$ exists and equals $1 / b^{*}(x, y)$. This verifies "Hypothesis A" in [12] for convex bodies $K \subset \mathbb{R}^{n}$; i.e., the inscribed ellipse method and the pluripotential-theoretic method for obtaining Bernstein-Markov type estimates for convex bodies are equivalent.

Let $K$ be an arbitrary convex body in $\mathbb{R}^{n}$. Fix $x \in K^{o}$ and $y \in \mathbb{R}^{n} \backslash\{0\}$. Take a $b$-maximal ellipse $\mathcal{E}$ through $x$ with tangent direction $y$ at $x$. We will normalize and assume that $y$ is a unit vector; moreover, it will be convenient to have the center at $a$ instead of $x-a$. Thus we write

$$
\theta \rightarrow r(\theta)=(x-a) \cos \theta+b^{*}(x, y) y \sin \theta+a, \theta \in[0,2 \pi] .
$$

This is an $a$-maximal ellipse $\mathcal{E}$ by Proposition 3.2 i.e., $\mathcal{E}$ forms the real points of a leaf $L$,

$$
f(\zeta)=(x-a)\left[\frac{1}{2}(\zeta+1 / \zeta)\right]+b^{*}(x, y) y\left[\frac{i}{2}(\zeta-1 / \zeta)\right]+a,|\zeta| \geq 1,
$$

of our foliation for the extremal function $V_{K}$. We can compare this " $b$-maximal" form of the leaf with its $a$-maximal form (2.2) (with $\zeta$ replaced by $1 / \zeta$ ):

$$
f(\zeta)=A+c \zeta+\bar{c} / \zeta,|\zeta| \geq 1,
$$

where, for simplicity, we write $c:=\rho C$ in (2.2). Thus, from (2.3), $V_{K}(f(\zeta))=\log |\zeta|$ for $|\zeta| \geq 1$.

We first show that

$$
\lim _{r \rightarrow 1^{+}} \frac{f(r)-f(1)}{r-1}=i b^{*}(x, y) y .
$$

This follows from the calculation

$$
f(r)-f(1)=(x-a)\left(\frac{(r-1)^{2}}{2 r}\right)+i b^{*}(x, y) y \frac{(r-1)(r+1)}{2 r} .
$$


Thus the real tangent vector to the real curve $r \rightarrow f(r), r \geq 1$ as $r \rightarrow 1^{+}$is in the direction $i b^{*}(x, y) y$. Now $f(1)=x$ and $x \in K$, so $V_{K}(f(1))=V_{K}(x)=0$; since $f$ is a leaf of our foliation, $V_{K}(f(r))=\log r$. Hence

$$
\frac{V_{K}(f(r))-V_{K}(f(1))}{r-1}=\frac{\log r}{r-1} .
$$

This elementary calculation shows that for any convex body $K \subset \mathbb{R}^{n}$,

$$
\lim _{r \rightarrow 1^{+}} \frac{V_{K}(f(r))-V_{K}(f(1))}{b^{*}(x, y)(r-1)}=\frac{1}{b^{*}(x, y)}
$$

i.e., the curvilinear limit along the curve $f(r)$ in the direction of $i y$ at $x$ exists and equals $\frac{1}{b^{*}(x, y)}$.

Note that

$$
f(r)-f(1)=f(r)-x=i b^{*}(x, y) y(r-1)+0\left((r-1)^{2}\right),
$$

so that the point $x+i b^{*}(x, y) y(r-1)$ is $O\left((r-1)^{2}\right)$ close to the point $f(r)$. We use the explicit form (4.3) of the leaf to verify the existence of the limit in the directional derivative $\delta_{B}(x, y)$.

Theorem 4.1. Let $K$ be a convex body in $\mathbb{R}^{n}$. Then the limit in the definition of the directional derivative exists and equals $\frac{1}{b^{*}(x, y)}$ :

$$
\delta_{B}(x, y):=\lim _{t \rightarrow 0^{+}} \frac{V_{K}(x+i t y)}{t}=\frac{1}{b^{*}(x, y)} .
$$

Proof. If we can show

$$
\lim _{r \rightarrow 1^{+}} \frac{V_{K}(f(r))-V_{K}\left(x+i b^{*}(x, y) y(r-1)\right)}{b^{*}(x, y)(r-1)}=0,
$$

then using (4.5) and the preceeding discussion, we will have the result.

We first consider the case when $K$ admits a continuous foliation; i.e., $K \in \mathcal{C}$. Consider a fixed point $w:=x+i b^{*}(x, y) y(r-1) \in \mathbb{C}^{n}$. This belongs to some foliation leaf $M$, which we write in the form (4.4):

$$
g(\zeta)=\alpha+\gamma \zeta+\bar{\gamma} / \zeta: \mathbb{C} \backslash \Delta \rightarrow M \subset \mathbb{C}^{n} .
$$

We need to use the facts that when $r \rightarrow 1^{+}$, then $w \rightarrow x \in L$, and, by continuity of the foliation, the leaf parameters for $(g, M)$ should converge to those of $(f, L)$; i.e., $\alpha \rightarrow A$ and $\gamma \rightarrow c$. We remark that if we compare (4.3) and (4.4), writing $b:=b^{*}(x, y)$ we have the relations

$$
A=a \text { and } c=\frac{1}{2}(x-a+i b y) .
$$

Here we suppress a rotational invariance: the substitution $\zeta^{\prime}:=\zeta e^{i \varphi}$ for any fixed constant $\varphi$ describes the same leaf with a different parametrization; thus we fix its value so that

$$
\xi:=g(1)=2 \operatorname{Re} \gamma+\alpha
$$

is closest to $x:=f(1)=2 \operatorname{Re} c+A$, i.e., $|g(1)-f(1)| \leq\left|g\left(e^{i \theta}\right)-f(1)\right|$ for all $\theta$. To emphasize, writing the leaf $(f, L)$ in $b$-maximal form (4.3),

$$
f(\zeta)=(x-a) \frac{1}{2}\left(\zeta+\frac{1}{\zeta}\right)+b y \frac{i}{2}\left(\zeta-\frac{1}{\zeta}\right)+a,
$$


where, from (4.8) and the fact that $y$ is a unit vector, $b:=2|\operatorname{Im} c|>0$ and $y:=$ $\frac{2}{b} \operatorname{Im} c \in \mathbb{R}^{n}$. Now, a priori, we do not know if $(g, M)$ is $b$-maximal (a posteriori, it is; see Corollary 4.3). However, we may still write this leaf in the form

$$
g(\zeta)=(\xi-\alpha) \frac{1}{2}\left(\zeta+\frac{1}{\zeta}\right)+\beta \eta \frac{i}{2}\left(\zeta-\frac{1}{\zeta}\right)+\alpha
$$

with $\beta:=2|\operatorname{Im} \gamma|>0$ and $\eta:=\frac{2}{\beta} \operatorname{Im} \gamma \in \mathbb{R}^{n}$. Note that continuity of the foliation implies $\beta>0$ since $b>0$; indeed, $\beta \sim b, \xi \sim x, \eta \sim y, \alpha \sim a$, and $\gamma \sim c$.

Since $w \in M$, there is a point $\omega \in \mathbb{C} \backslash \Delta$ with $g(\omega)=w$. Our task is to calculate $V_{K}(w)=V_{K}(g(\omega))$. On a leaf of the foliation we have the formula $V_{K}(g(\omega))=$ $\log |\omega|$, so it suffices to compute $\log |\omega|$. The representation of $w$ as $g(\omega)$ means that for $j=1, \ldots, n$,

$$
x_{j}+i b y_{j}(r-1)=w_{j}=g_{j}(\omega)=\left(\xi_{j}-\alpha_{j}\right) \frac{1}{2}\left(\omega+\frac{1}{\omega}\right)+\beta \eta_{j} \frac{i}{2}\left(\omega-\frac{1}{\omega}\right)+\alpha_{j} .
$$

Since $y$ and $\eta$ are unit vectors which are close to each other, we can choose a coordinate $j$ with $y_{j} \neq 0, \eta_{j} \neq 0$. For this coordinate $j$, the previous displayed equation gives

$$
\frac{1}{2}\left(\xi_{j}-\alpha_{j}+i \beta \eta_{j}\right) \omega^{2}+\left(\alpha_{j}-x_{j}-i b y_{j}(r-1)\right) \omega+\frac{1}{2}\left(\xi_{j}-\alpha_{j}-i \beta \eta_{j}\right)=0,
$$

a quadratic equation in $\omega$. Corresponding to the double mapping properties of the Joukowski map $\frac{1}{2}(\zeta+1 / \zeta)$, there are two roots, one in $|\zeta|<1$ and one in $|\zeta|>1$, the latter being our $\omega$ as we considered the mapping of the exterior of the unit disc $\Delta$. For convenience, put $\rho:=b(r-1) y_{j}$. Since $b y_{j} \neq 0, \rho \asymp r-1$. By the quadratic formula,

$$
\omega_{1,2}=\frac{x_{j}-\alpha_{j}+i \rho \pm \sqrt{\left(\alpha_{j}-x_{j}-i \rho\right)^{2}-\left(\xi_{j}-\alpha_{j}\right)^{2}-\beta^{2} \eta_{j}^{2}}}{\xi_{j}-\alpha_{j}+i \beta \eta_{j}} .
$$

Set $Q:=\beta^{2} \eta_{j}^{2}+\left(\xi_{j}-\alpha_{j}\right)^{2}-\left(x_{j}-\alpha_{j}\right)^{2} \sim b^{2} y_{j}^{2}>0$ by continuity of the leaf parameters and choice of $j$. Using this and the simple formula $\sqrt{A+2 B}=$ $\sqrt{A}+B / \sqrt{A}+O\left(B^{2} / A^{3 / 2}\right)$, valid uniformly for $|B|<A / 3$, say, we can rewrite the square root as

$$
\begin{aligned}
& \pm \sqrt{\left(x_{j}-\alpha_{j}\right)^{2}-i 2\left(\alpha_{j}-x_{j}\right) \rho-\rho^{2}-\left(\xi_{j}-\alpha_{j}\right)^{2}-\beta^{2} \eta_{j}^{2}} \\
& \quad= \pm\left\{\frac{\left(x_{j}-\alpha_{j}\right) \rho}{\sqrt{Q}}+i \sqrt{Q}+O\left(\rho^{2}\right)\right\} .
\end{aligned}
$$

Put $P:=\xi_{j}-\alpha_{j}+i \beta \eta_{j}$. Then

$$
\begin{aligned}
\left|\omega_{1,2} P\right|^{2} & =\left|\left(x_{j}-\alpha_{j}\right)\left(1 \pm \frac{\rho}{\sqrt{Q}}\right)+i[ \pm \sqrt{Q}+\rho]+O\left(\rho^{2}\right)\right|^{2} \\
& =\left(\alpha_{j}-x_{j}\right)^{2}+Q \pm \frac{2 \rho}{\sqrt{Q}}\left(Q+\left(\alpha_{j}-x_{j}\right)^{2}\right)+O\left(\rho^{2}\right) .
\end{aligned}
$$

We have the identity $|P|^{2}=\left(\alpha_{j}-x_{j}\right)^{2}+Q$; dividing by this quantity on both sides yields

$$
\left|\omega_{1,2}\right|^{2}=1 \pm \frac{2 \rho}{\sqrt{Q}}+O\left(\rho^{2}\right) .
$$


Fixing the branch of the square root with $\sqrt{Q}>0$, it is clear that among the choice of signs in \pm the one equal to the sign of $y_{j}$ leads to the larger absolute value (and the one with $|\omega|$ exceeding 1); hence for such $\omega$ with $g(\omega)=w$,

$$
|\omega|^{2}=1+\frac{2|\rho|}{\sqrt{Q}}+O\left(\rho^{2}\right)=1+\frac{2 b\left|y_{j}\right|(r-1)}{\sqrt{Q}}+O\left((r-1)^{2}\right)
$$

so that

$$
\log |\omega|^{2}=\frac{2 b\left|y_{j}\right|(r-1)}{\sqrt{Q}}+O\left((r-1)^{2}\right)
$$

Hence

$$
\frac{V_{K}(x+i b y(r-1))}{r-1}=\frac{V_{K}(g(\omega))}{r-1}=\frac{\log |\omega|}{r-1}=\frac{1}{2} \frac{\log |\omega|^{2}}{r-1}=\frac{b\left|y_{j}\right|}{\sqrt{Q}}+O(r-1) .
$$

From the continuity of the foliation, as $r \rightarrow 1$ we have $\xi_{j} \rightarrow x_{j}, \alpha_{j} \rightarrow a_{j}, \beta \rightarrow b$, and thus $\sqrt{Q} \rightarrow b\left|y_{j}\right|$. Hence

$$
\lim _{r \rightarrow 1^{+}} \frac{V_{K}(x+i b y(r-1))}{r-1}=1=\lim _{r \rightarrow 1^{+}} \frac{V_{K}(f(r))}{r-1} .
$$

This verifies (4.7) and hence (4.6) in the case when $K$ admits a continuous foliation.

For a general convex body $K$, we use the previous case and an appropriate approximation argument to verify (4.6). To emphasize the set(s) under discussion, we write

$$
b^{*}(K ; x, y):=b^{*}(x, y) \text { for the set } K .
$$

We need to prove two inequalities:

$$
\frac{1}{b^{*}(K ; x, y)} \leq \liminf _{t \rightarrow 0+} \frac{V_{K}(x+i t y)}{t}
$$

and

$$
\frac{1}{b^{*}(K ; x, y)} \geq \limsup _{t \rightarrow 0+} \frac{V_{K}(x+i t y)}{t} .
$$

Note that if $K$ and $\kappa$ are two convex bodies, we have for any $y \in \mathbb{R}^{n}$ the inequalities

$$
b^{*}(K ; x, y)\left\{\begin{array}{lll}
\leq b^{*}(\kappa ; x, y) & \text { if } & K \subset \kappa, \\
\geq b^{*}(\kappa ; x, y) & \text { if } & \kappa \subset K,
\end{array}\right.
$$

and for any $y \in \mathbb{R}^{n}$ and any $t \in \mathbb{R}$ the inequalities

$$
V_{K}(x+i t y)\left\{\begin{array}{lll}
\geq V_{\kappa}(x+i t y) & \text { if } & K \subset \kappa, \\
\leq V_{\kappa}(x+i t y) & \text { if } & \kappa \subset K
\end{array}\right.
$$

Fix $\alpha<1$ arbitrarily close to 1 and choose $\delta>0$ small (to be determined later in terms of $\alpha$ ). From the discussion at the end of section $2, \mathcal{C}$ is dense in $\mathcal{K}$; thus we can find $\kappa \in \mathcal{C}$ such that the Hausdorff distance between $\kappa$ and $K$ is at most $\delta$. Take an $\alpha$-dilated (at $x$ ) copy $K_{1}$ of $K$ and an $\alpha$-dilated copy $\kappa_{1}$ of $\kappa$. Then $x \in K_{1}^{0}$, and if $\delta=\delta(\alpha)$ is sufficiently small, we have $\kappa_{1} \subset K$. We also take the $1 / \alpha$-dilated copies $K_{2}$ and $\kappa_{2}$ of $K$ and $\kappa$. Again for small enough $\delta$, we will have $K \subset \kappa_{2}$. Note that $\kappa_{2}$ is the $\alpha^{-2}$-dilated copy of $\kappa_{1}$; hence $b^{*}\left(\kappa_{2} ; x, y\right)=\alpha^{-2} b^{*}\left(\kappa_{1} ; x, y\right)$. 
Therefore, using (4.6) for $\kappa_{1}$ and $\kappa_{2}$, we obtain

$$
\lim _{t \rightarrow 0+} \frac{V_{\kappa_{2}}(x+i t y)}{t}=\frac{1}{b^{*}\left(\kappa_{2} ; x, y\right)}=\alpha^{2} \frac{1}{b^{*}\left(\kappa_{1} ; x, y\right)}=\alpha^{2} \lim _{t \rightarrow 0+} \frac{V_{\kappa_{1}}(x+i t y)}{t} .
$$

Using (4.13), (4.11) and (4.12), we obtain

$$
\begin{aligned}
\frac{1}{b^{*}(K ; x, y)} & \leq \frac{1}{b^{*}\left(\kappa_{1} ; x, y\right)}=\lim _{t \rightarrow 0+} \frac{V_{\kappa_{1}}(x+i t y)}{t} \\
& =\frac{1}{\alpha^{2}} \lim _{t \rightarrow 0+} \frac{V_{\kappa_{2}}(x+i t y)}{t}= \\
& \leq \frac{1}{\alpha^{2}} \liminf _{t \rightarrow 0+} \frac{V_{K}(x+i t y)}{t} ;
\end{aligned}
$$

in a similar fashion we get, interchanging the roles of $\kappa_{1}$ and $\kappa_{2}$,

$$
\frac{1}{b^{*}(K ; x, y)} \geq \frac{1}{b^{*}\left(\kappa_{2} ; x, y\right)} \geq \alpha^{2} \limsup _{t \rightarrow 0+} \frac{V_{K}(x+i t y)}{t} .
$$

Since $\alpha$ can be taken arbitrarily close to 1 , (4.9) and (4.10) follow from (4.14) and (4.15).

Remark 4.2. Observe that the essential property used to verify (4.7) for $K \in \mathcal{C}$ is (2.3), i.e., that $V_{K}(f(\zeta))=\log |\zeta|$ on $L$ (i.e., for $|\zeta| \geq 1$ ), which is equivalent to the $a$-maximality of the real ellipse $\mathcal{E} \subset L$ (see section 2).

Corollary 4.3. For all $x \in K^{o}$ and $y \in T_{x} \mathcal{E}$, for any convex body $K$, an ellipse $\mathcal{E} \subset K$ is a-maximal if and only if it is $b$-maximal.

Proof. That $b$-maximality implies $a$-maximality was proved in Proposition 3.2 , For the converse, we first suppose that $K \in \mathcal{C}$. Let $\mathcal{E}$ be an $a$-maximal ellipse. Fix $x \in K^{o}$ and $y \in T_{x} \mathcal{E}$ a unit vector. Then $\mathcal{E}=f(\partial \Delta)$, where

$$
f(\zeta)=(x-\alpha) \frac{1}{2}\left(\zeta+\frac{1}{\zeta}\right)+\beta y \frac{i}{2}\left(\zeta-\frac{1}{\zeta}\right)+\alpha
$$

and $V_{K}(f(\zeta))=\log |\zeta|$ for $|\zeta| \geq 1$, so that, from the remark,

$$
1 / \beta=\lim _{r \rightarrow 1^{+}} \frac{V_{K}(f(r))}{\beta(r-1)}=\lim _{r \rightarrow 1^{+}} \frac{V_{K}(x+i \beta y(r-1))}{\beta(r-1)} .
$$

Writing $t=\beta(r-1)$ in the limit on the right and using Theorem 4.1

$$
1 / \beta=\lim _{t \rightarrow 0^{+}} \frac{V_{K}(x+i t y)}{t}=\frac{1}{b^{*}(x, y)}
$$

so that $\mathcal{E}$ is $b$-maximal for $x, y$.

Now let $K \in \mathcal{K}$ be an arbitrary convex body. We consider first the case where $\mathcal{E}$ is the unique $a$-maximal ellipse for $[c] \in H$, i.e., for its orientation and eccentricity, and we again write $\mathcal{E}=f(\partial \Delta)$ as in (4.16). Take a sequence $\left\{K_{j}\right\} \subset \mathcal{C}$ with $K_{j} \searrow K$. For each $j$, let $\mathcal{E}_{j}$ be the unique $a$-maximal ellipse for $K_{j}$, [c], and let $f_{j}$ denote the corresponding leaf. Then (cf. [7]) $f_{j} \rightarrow f$ uniformly so that $\mathcal{E}_{j} \rightarrow \mathcal{E}$. As in the proof of Theorem 4.1, we may write

$$
f_{j}(\zeta)=\left(x_{j}-\alpha_{j}\right) \frac{1}{2}\left(\zeta+\frac{1}{\zeta}\right)+\beta_{j} y_{j} \frac{i}{2}\left(\zeta-\frac{1}{\zeta}\right)+\alpha_{j} .
$$


By the first part of the proof, $\mathcal{E}_{j}$ is $b$-maximal in $K_{j}$, so that $\beta_{j}=b^{*}\left(K_{j} ; x_{j}, y_{j}\right)$. The uniform convergence of $f_{j}$ to $f$ implies that $\alpha_{j} \rightarrow \alpha, x_{j} \rightarrow x$ and $y_{j} \rightarrow y$. Moreover, since $x \in K^{o}$, for $j$ sufficiently large, $x_{j} \in K^{o}$. From the continuity of $b^{*}$ (Proposition 3.3) and the fact that $K \subset K_{j}$,

$$
b^{*}(K ; x, y)=\lim _{j \rightarrow \infty} b^{*}\left(K ; x_{j}, y_{j}\right) \leq \lim _{j \rightarrow \infty} b^{*}\left(K_{j} ; x_{j}, y_{j}\right)=\lim _{j \rightarrow \infty} \beta_{j}=\beta .
$$

Hence $\beta=b^{*}(K ; x, y)$ and $\mathcal{E}$ is $b$-maximal.

In the case where $\mathcal{E}$ is not the unique $a$-maximal ellipse for the corresponding $[c] \in H$, it is an $a$-maximal ellipse for this $[c]$ and a "strip" $S$, i.e., a closed body $S$ bounded by two parallel hyperplanes $P_{1}, P_{2}$ with $K \subset S$ (see section 7 of [7]). If $\mathcal{E}$ is given by

$$
r(\theta)=f\left(e^{i \theta}\right)=(x-\alpha) \cos \theta+\beta y \sin \theta+\alpha,
$$

then there is $\theta_{0}$ such that $r\left(\theta_{0}\right) \in P_{1}$ and $r\left(\theta_{0}+\pi\right) \in P_{2}$. It is therefore sufficient to show that any ellipse $\mathcal{E} \subset K$ that intersects $P_{1}, P_{2}$ is $b$-maximal for any $x \in \mathcal{E} \cap K^{o}$, $y \in T_{x} \mathcal{E}$. Take a sufficiently large convex set $T$ that is symmetric about the center of the ellipse (e.g., a large box) so that $K \subset T \subset S$. Clearly $\mathcal{E}$ is $a$-maximal for $T$. Since $T$ is symmetric, $T \in \mathcal{C}$, so by the first part of the proof, $\mathcal{E}$ is $b$-maximal for $T, x, y$. Hence it is also $b$-maximal for $K, x, y$.

We turn to the Monge-Ampère measure. We know that $\left(d d^{c} V_{K}\right)^{n}$ is supported in $K$ and is absolutely continuous with respect to Lebesgue measure $d x$ on $K^{o}$; i.e., $\left(d d^{c} V_{K}\right)^{n}=\tilde{\lambda}(x) d x$ for a locally integrable non-negative function $\tilde{c}$ on $K^{o}[\underline{3}$. Baran has proved the following (see [2], Propositions 1.10, 1.11 and Lemma 1.12).

Proposition 4.4. Let $D \subset \mathbf{C}^{n}$ and let $\Omega:=D \cap \mathbb{R}^{n}$. Let $u$ be a non-negative psh function on $D$ which satisfies:

$$
\begin{aligned}
\text { i. } & \Omega=\{u=0\}, \\
\text { ii. } & \left(d d^{c} u\right)^{n}=0 \text { on } D \backslash \Omega, \\
\text { iii. } & \left(d d^{c} u\right)^{n}=\lambda(x) d x \text { on } \Omega \text { where } \lambda \in L_{\text {loc }}^{1}(\Omega), \\
\text { iv. } & \text { for all } x \in \Omega, y \in \mathbb{R}^{n}, \text { the limit } \\
& h(x, y):=\lim _{t \rightarrow 0^{+}} \frac{u(x+i t y)}{t} \text { exists and is continuous on } \Omega \times i \mathbb{R}^{n},
\end{aligned}
$$

v. for all $x \in \Omega, y \rightarrow h(x, y)$ is a norm.

Then

$$
\lambda(x)=n ! \operatorname{vol}\{y: h(x, y) \leq 1\}^{*}
$$

and $\lambda(x)$ is a continuous function on $\Omega$.

We now obtain the generalization of (1.3).

Corollary 4.5. Let $K$ be a convex body in $\mathbb{R}^{n}$. Then

$$
\left(d d^{c} V_{K}\right)^{n}=\lambda(x) d x \text { for } x \in K^{o},
$$

where $\lambda(x)=n ! \operatorname{vol}\left(\left\{y: \delta_{B}(x, y)=\frac{1}{b^{*}(x, y)} \leq 1\right\}^{*}\right)$ is continuous. Moreover, $\left(d d^{c} V_{K}\right)^{n}$ puts no mass on the boundary $\partial K$ (relative to $\left.\mathbb{R}^{n}\right)$.

Proof. The formula for $\left(d d^{c} V_{K}\right)^{n}$ on $K^{o}$ is immediate from Theorem 4.1. Proposition 4.4 and the paragraph preceding it, and Proposition 3.3 . To show that $\left(d d^{c} V_{K}\right)^{n}$ puts no mass on the boundary $\partial K$, we proceed as in $[3]$. Let $\left\{K_{j}\right\}$ be a sequence of convex bodies in $\mathbb{R}^{n}$ with real-analytic boundaries $\left\{\partial K_{j}\right\}$ such that $K_{j}$ 
increases to $K$. Then $\partial K_{j}$ is pluripolar so that $\left(d d^{c} V_{K_{j}}\right)^{n}$ puts no mass on $\partial K_{j}$ (cf. Proposition 4.6.4 of [8]). Writing $\left(d d^{c} V_{K_{j}}\right)^{n}:=\lambda_{j}(x) d x$ for $x \in K_{j}^{o}$, we have

$$
(2 \pi)^{n}=\int_{K_{j}^{o}} \lambda_{j}(x) d x=\int_{K^{o}} \lambda_{j}(x) d x,
$$

where we extend $\lambda_{j}(x)$ to be zero outside of $K_{j}^{o}$. Since $\int_{K^{o}} \lambda_{j}(x) d x=(2 \pi)^{n}<\infty$, and the density functions $\lambda_{j}(x)$ increase almost everywhere on $K^{o}$ to $\lambda(x)$ (cf. (4.11)), by dominated convergence we have

$$
(2 \pi)^{n}=\lim _{j \rightarrow \infty} \int_{K^{o}} \lambda_{j}(x) d x=\int_{K^{o}} \lambda(x) d x .
$$

Thus $\left(d d^{c} V_{K}\right)^{n}$ puts no mass on the boundary $\partial K$.

We end this note with a final remark on Bernstein-Markov inequalities. Baran [2] conjectured that we have equality $\delta_{M}(x, y)=\delta_{B}^{(i)}(x, y)$ in (4.1) for general convex bodies. With respect to this conjecture, we make the following observation: if we can prove $\delta_{M}(x, y)=\delta_{B}(x, y)$ for a triangle $T$ in $\mathbb{R}^{2}$, then equality holds for all convex bodies in $\mathbb{R}^{2}$. For let $K$ be a convex body in $\mathbb{R}^{2}$. Fix $x \in K^{o}$ and $|y|=1$. Take a $b$-maximal ellipse $\mathcal{E}=\mathcal{E}(x, y)$ for $K$ with parameter $b=b^{*}(x, y)$ and, as in (1) or (2) at the end of section 3 , take a rectangle or triangle $T$ containing $K$ in which $\mathcal{E}$ is an $a$-maximal ellipse. Then since $\delta_{B}^{T}(x, y)=\frac{1}{b^{*}(x, y)}=\delta_{B}^{K}(x, y)$, we have

$$
\delta_{M}^{K}(x, y) \geq \delta_{M}^{T}(x, y)=\delta_{B}^{T}(x, y)=\delta_{B}^{K}(x, y) .
$$

From (4.1), $\delta_{M}^{K}(x, y) \leq \delta_{B}^{K}(x, y)$ and equality holds.

\section{REFERENCES}

[1] M. Baran, Plurisubharmonic extremal functions and complex foliations for the complement of convex sets in $\mathbb{R}^{n}$, Michigan Math. J. 39 (1992), 395-404. MR.1182495 (93j:32022)

[2] M. Baran, Complex equilibrium measure and Bernstein type theorems for compact sets in $\mathbb{R}^{n}$, Proc. AMS 123 (1995), no. 2, 485-494. MR.1219719(95c:31006)

[3] E. Bedford and B. A. Taylor, The complex equilibrium measure of a symmetric convex set in $\mathbb{R}^{n}$, Trans. AMS 294 (1986), 705-717. MR825731 (87f:32039)

[4] P. Borwein and T. Erdélyi, Polynomials and polynomial inequalities, Graduate Texts in Mathematics, Springer-Verlag, 1995. MR1367960(97e:41001)

[5] L. Bos, N. Levenberg and S. Waldron, Pseudometrics, distances, and multivariate polynomial inequalities, Journal of Approximation Theory 153 (2008), no. 1, 80-96. MR2432555 (2009d:41015)

[6] D. Burns, N. Levenberg and S. Ma'u, Pluripotential theory for convex bodies in $\mathbb{R}^{N}$, Math. Zeitschrift 250 (2005), no. 1, 91-111. MR2136404 (2005k:32044)

[7] _ Exterior Monge-Ampère Solutions, Adv. Math. 222 (2009), 331-358. MR 2538012 (2010g:32062)

[8] M. Klimek, Pluripotential Theory, Clarendon Press, Oxford, 1991. MR.1150978 (93h:32021)

[9] M. Lundin, The extremal plurisubharmonic function for the complement of the disk in $\mathbb{R}^{2}$, unpublished preprint, 1984.

[10] _ The extremal plurisubharmonic function for the complement of convex, symmetric subsets of $\mathbb{R}^{n}$, Michigan Math. J. 32 (1985), 197-201. MR.783573 (86h:32030)

[11] L. Milev and Sz. Révész, Bernstein's inequality for multivariate polynomials on the standard simplex, J. Inequal. Appl. (2005), no. 2, 145-163. MR2173358(2006g:41026)

[12] Sz. Révész, A comparative analysis of Bernstein type estimates for the derivative of multivariate polynomials, Ann. Polon. Math. 88 (2006), no. 3, 229-245. MR2260403(2007f:41012) 
[13] Sz. Révész and Y. Sarantopoulos, A generalized Minkowski functional with applications in approximation theory, J. Convex Analysis 11 (2004), no. 2, 303-334. MR2158907|(2006e:52001)

[14] Y. Sarantopoulos, Bounds on the derivatives of polynomials on Banach spaces, Math. Proc. Camb. Phil. Soc. 110 (1991), 307-312. MR1113429 (92j:46084)

Department of Mathematics, University of Michigan, Ann Arbor, Michigan 481091043

E-mail address: dburns@umich.edu

Department of Mathematics, Indiana University, Bloomington, Indiana 47405

E-mail address: nlevenbe@indiana.edu

Mathematics Division, University of the South Pacific, SCiMS, Suva, Fiji

E-mail address: mau_s@usp.ac.fj

A. Rényi Institute of Mathematics, Hungarian Academy of Sciences, Budapest, P.O.B. 127, 1364 Hungary

E-mail address: revesz@renyi.hu 